\title{
A System Architecture for a Context-aware Blended Mobile Learning Environment
}

\author{
Ivica Boticki ${ }^{1}$, Natasa Hoic-Bozic ${ }^{2}$ and Ivan Budiscak ${ }^{1}$ \\ ${ }^{1}$ Faculty of Electrical Engineering and Computing, University of Zagreb, Croatia \\ 2 Department of Informatics, University of Rijeka, Croatia
}

\begin{abstract}
Mobility offers new possibilities for interaction and communication in education. Pervasive nature of mobile devices makes learning on the move and across time a reality. Their characteristics fit into the blended use of technology in education since they posses socializing powers, re-conceptualize learning by making it more informal, ambient, collaborative and personal. Nevertheless, the problem of creation and coordination of a learning environment based on mobile devices still exists. This article presents a system called MILE and its extensible context-aware architecture which supports the use of mobile devices in blended learning environments. In the end, some experience and lessons learned during the development of the system are described.
\end{abstract}

Keywords: mobile learning, architectures for educational technology systems, interactive learning environments, context-aware learning

\section{Introduction}

In addition to being less formal and lifelong, learning in the new era shifts from theoretical to practical and integrated, from remembering facts to understanding and being critically aware and from face-to-face to distance and e-learning [14]. The information technology, therefore, has to be used to facilitate learning and it should, according to the blended learning paradigm, be used as just one of many elements facilitating quality learning [39].

Mobile computing, referred to by some authors as MoCo [11], is used in business, everyday life and education. It "describes any technology that enables people to access information and which supports them in daily workflows independent of location" [12]. Miniature mobile devices, ever-rising power and possibilities of communication (e.g. WiFi, 3G etc.) support an area of human activity that has long been technologically neglected: the life on the move. In addition to being used by individuals located out of their living and working environments, mobile technology is used by certain professions in order to acquire contextualized knowledge while on the move [17], [12], [2].

In addition to various everyday human activities, mobile devices are said to achieve largescale impact on learning [31]. They support elearning by making it less dependent of the location, more contextual and pedagogically more oriented to the constructivist and situated approaches to learning [20], [27].

In order to support mobile learners in their contexts the system MILE (Mobile and Interactive Learning Environment) has been designed. The main purpose of this system is to support the didactic and the discursive approach to learning [17]. Students equipped with mobile devices of various kinds (laptops, tablets, PDAs and mobile phones), are connected to the system via faculty or campus wireless networks. They benefit from the central server component which distributes learning-related events to the tools available as a client side MILE application.

MILE's architecture is designed specifically by having mobile learners in mind. Users are not overwhelmed with technological tasks, such as the configuration of the system, filling in server 
addresses etc., since the system takes care of these in an intelligent way. Furthermore, a core component of the system, context engine, was built to take into account users' context and adapt system's behavior according to it. To achieve that, context engine utilizes concepts of subscriptions and contextually triggered actions.

Students using the system benefit from various tools, called modules, whose main aim is to enhance learning by enticing interaction and collaboration in a blended approach to learning. Some of the modules are MCollaboration, MNotebook and MWhiteboard and can be used in everyday classroom to support various educational activities.

The remainder of the paper explains the terms mobile learning and context-aware mobile learning and presents system architecture and applicative modules of the MILE system together with the way they can be used in class to support learning activities. In the end, some experience and lessons learned during the development of the system are presented.

\section{Mobile Learning}

Although many debates on the appropriateness of use of mobile devices in education are being led, theorists agree on at least one thing: they strongly support "learning anywhere anytime" paradigm [35] long promoted by the elearning society. Learning on the move attracts researchers since it has found a way of better using an invaluable resource: the time when a person is mobile [22].

For mobile learning to occur, a person does not have to actually move while learning. It is sufficient that he or she is out of her standard learning environment without access to static learning resources (e.g. libraries, desktop computers, textbooks etc.) [34], [35]. Mobile learning is ambient, that is aware of the surroundings in which it occurs, personal since mobile devices are personal belongings and informal because it occurs whenever and wherever the need exists [34].

Furthermore, mobility could lead to the transformation of education towards a more student- oriented, facilitator-oriented, informal and problem-based [36], [28]. The shift in the educational process is strongly supported by technology and reflects the competences required by the today's global society.

The main idea of the MILE system is to support mobile learners, both in the didactic and discursive approach to learning, in multiple pedagogical approaches to learning. Behaviorally, teachers can utilize it to get instant feedback on the matter presented, while students benefit from its didactical capabilities when browsing through the learning material in the classroom or on the move.

Some components of the system are created to support cognitive and socio-constructivistic pedagogical approaches to learning [38]. MILE's tools can be used to construct knowledge of higher order concepts and to support situated approach to learning through collaboration on group tasks as well as communities of practice.

\subsection{Context-aware Mobile Learning}

Context-aware mobile applications adapt to their users and, in a way, sense their needs [40]. Applications for mobile devices should find the way to appropriately represent complex information and convey it to their users in an efficient manner [1].

Context is referred to as the information that applications should use to automatically adapt to their users [4], [5], [10]. Some more complex definition were given by Day and Abowd [7]: "Context is any information that can be used to characterize the situation of entities (whether a person, place or object) that are considered relevant to the interaction between a user and an application, including the user and the application themselves" and by Brown [3]: "Context are the elements of the user's environment which the computer knows about".

The most important contextual information are said to be location, environment, identity and time. Location as a part of context is considered to be the most important since much other contextual information can be inferred from it. By knowing user's location, he or she can be automatically related to the environment and other 
users. In addition to that, the time component of context can help in sorting spatial information so that it can be seen as a sequence of events [7]. Although location, time and identity represent, according to some authors [4], [5], [10], [7], 99\% of needed contextual information, sensory information, such as light intensity, noise etc. can also be used to give a more detailed context representation.

The art of constructing systems which use contextual information is often called context-awareness. Context-awareness should be an important element of technology-supported learning, especially when mobile learning is considered [5]. Context-awareness does not only make applications more intuitive and user-friendly, it opens up many new possibilities when enhancement of classical learning environments is concerned. It could be integrated with pedagogical approaches, such as collaborative learning, problem-based learning, situated learning etc. [40].

Context-awareness is a first class concept when MILE's architecture is considered. Contextual information is used to enhance and facilitate various educational activities supported by the system. Following Wang's definition of CAML (context aware mobile learning) [40], MILE possesses basic contextual dimensions called identity, spatio-temporal and facility dimension. These form the base for the generation of other, higher-order contextual dimensions. Identity is used to identify users; WiFi-based location-awareness to provide system and users with location-enhanced services while spatiotemporal dimension and facility dimension are used to fine-tune system's technical characteristics such as devices' display orientation and size.

In order to support collaborative activities, the activity and community dimensions of contextaware mobile learning are utilized. Student activity is being aggregated, primarily to support collaborative activities. Community dimension is used to support pedagogical activities in which students form ad hoc learning groups. Community dimension of CAML in the MILE system can be used to support situated approach to learning (e.g. communities of practice).
Context triggered events [40] are used throughout the system to support communication and to provide a base for the development of new services. To disseminate events to clients, a special means of communication called distributed event protocol has been designed.

\section{Related Work}

From the technical point of view, there are many projects dealing with mobile learning ranging from simple ones, utilizing mobile devices with preinstalled software to support all sorts of learning activities such as learning foreign languages via SMS [18] or reading e-books [16] to more complex custom made software usually aimed at supporting a specific learning activity such as museum tours [29], [13], fieldwork [26] or medicine [12], [37]. There are some quality reports summarizing projects and activities in the field of m-learning [17], [6].

In addition to the hardware and software requirements, mobile learning systems can be evaluated by the pedagogical principles they use. They can be purely behavioral, cognitively oriented or designed to support situated approach to learning [22]. Although the relationship between the technical and pedagogical side cannot be firmly established, it seems that the support for the cognitive and situated approaches to mobile learning requires increased technical complexity when software is concerned [17].

MILE's key characteristic is a specifically designed system architecture which uses contextawareness and wireless communication to support various mobile learning activities. There are some accounts of software using contextawareness, collaboration and virtual environments to support learning. Some of them discuss systems that facilitate educational information sources [32], others deal with mobile support for problem-based learning [19], discuss virtual learning environments with locationaware services [21], [3] or are focused on campuswide initiatives [9]. They were used as a starting point when the development of MILE's extensible, modular and platform independent architecture was considered. 


\section{A System Architecture for a Context-aware Mobile Blended Learning Environment}

\subsection{MILE - Mobile and Interactive Learning Environment}

The main effort of the project is to introduce mobile devices into an everyday educational setting in order to improve interaction and collaboration. MILE takes a form of a distributed learning network [15], [17] and uses mobility to support distributed learning activities in a blended learning approach. It is used by both students and teachers as depicted in Figure 1.

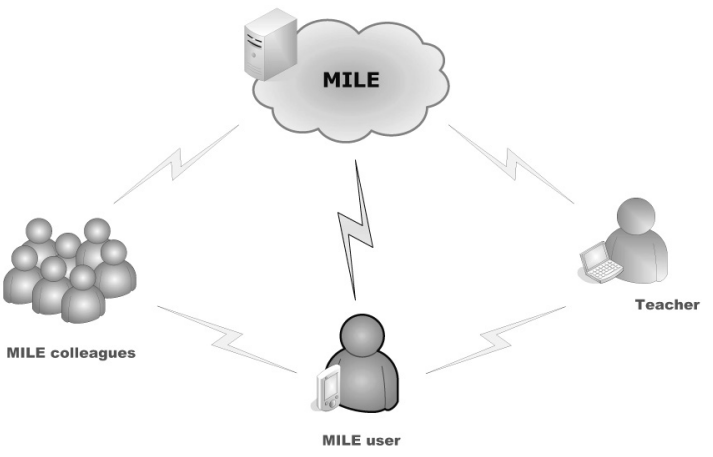

Figure 1. MILE system and its main actors.

Mobile learning community believes that mobile technologies and software for mobile devices should not be appropriated to satisfy the needs of mobile learners. Instead of that, applications should be built from ground up, in order to better fit the learners and educational process [15].

Technically speaking, MILE consists of two distinct parts: MILE framework, which can be seen as a common system infrastructure, and applicative modules used by learners.

\subsection{System Architecture of the MILE System}

Based on the requirements stated in the previous chapters, the system architecture was designed in order to provide a solid ground for the development of various applicative modules for mobile learning.

MILE is a distributed and service-oriented [25] system with its most important services being:

- Core services - used to manage contextual information and disseminate events to mobile users.

- System Services - used to provide services to mobile clients: ZeroConfigurationService (saves mobile users from gruelling configuration tasks), AuthentificationService, IPRegistrarService (manages active mobile clients) and ContextService (gathers client contextual information).

- Module services - services providing specific functionality to the applicative modules and mobile clients.

For service implementation Microsoft Windows Communication Foundation was used, which enabled the use of both TCP and HTTP protocols for the communication with server services [23].

In addition to its service orientation, MILE has a layered structure consisting of two distinct parts:

- MILE Framework (left hand side of the Figure 2) which includes desktop, device and server framework and is used by host applications and the event engine.

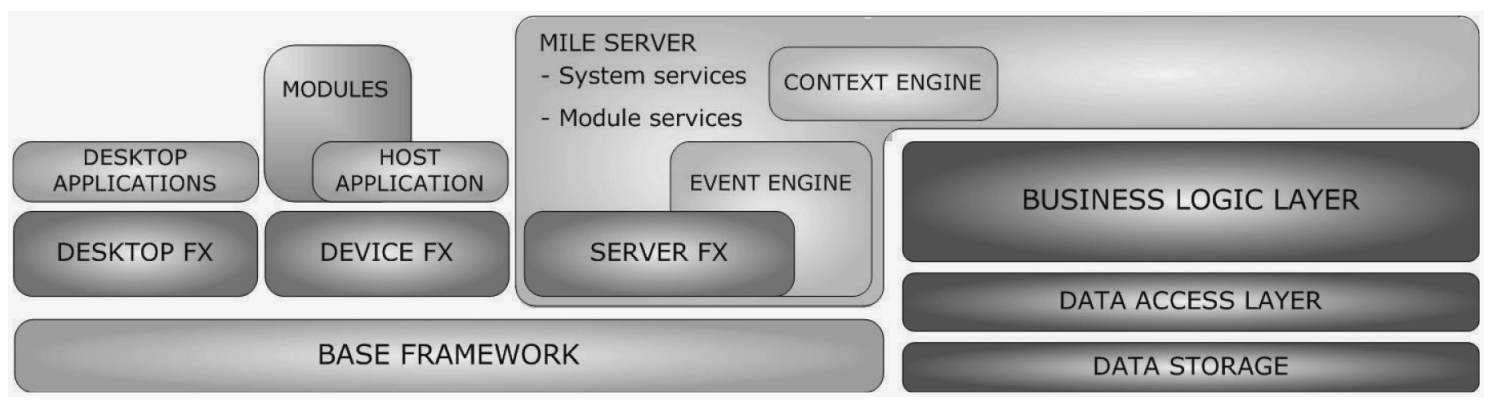

Figure 2. MILE framework stack. 
- MILE data access layer and business logic layer (right hand side of the Figure 2) provide common interface to system's data.

\subsection{The Communication Model}

The communication model is based on a clientserver architecture in which communication occurs over a TCP/IP network. Clients consume services provided by the server while the server initializes communication towards the clients using custom-made Distributed Events Protocol (DEP) (Figure 3).

The communication between a client and the server is not direct and is managed by the MILE framework. MILE framework consists of two major parts, the Server Framework and the Client Framework which is platform specific. This means that a client framework has to be developed for each platform, such as a mobile device (i.e. PocketPC) or a laptop computer, separately.

The server uses DEP (Distributed Event Protocol) to the communicate with one or more clients simultaneously. This is a one-way communication in which the server sends information about system events to its clients. These distributed events are then manifested on the client side as .NET events [8].

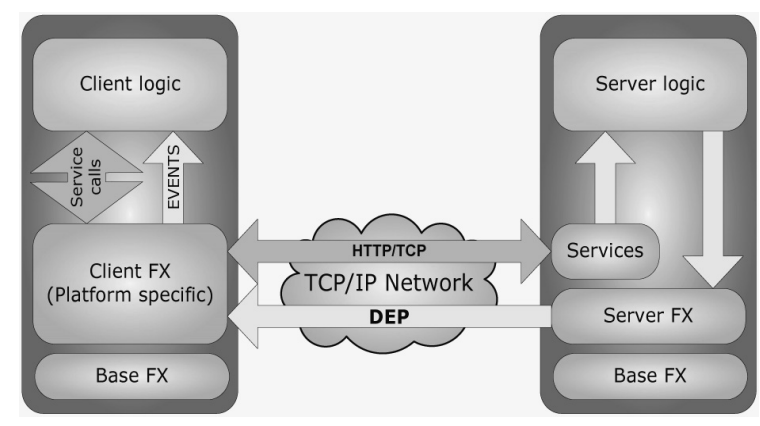

Figure 3. MILE communication model.

\subsection{The Server}

The Server Framework (Figure 2) plays an important role in achieving system functionality since it interprets all information gathered by the system.

The Server Framework has two main components: the Event Engine and the Context Engine and it uses two server services: the Subscription Service and the Context Service which collect all the information that needs to be contextually interpreted.

\subsubsection{The Context Service}

The Context Service collects contextual information from the clients; i.e. client's current location, temperature at current location, noise level, light intensity etc. One might assume that a client would need an attached sensor or a similar device to provide the system with such sort of information. To avoid that, a support for sensors that act as clients, in which a client (sensor) is used only to collect contextual information from the environment and to transfer it to the server, was developed.

\subsubsection{The Subscription Service}

The Subscription Service is used by clients to submit subscriptions. A subscription consists of two parts: a context which must be satisfied and an action that will be preformed if the context is satisfied. The term action actually refers to an event sent to the subscribed clients.

\subsection{Clients}

Basic idea behind the Client Framework is to provide services to client applications. In addition to that, its important function is the manifestation of distributed events as .NET events on the client side.

Event handling begins in the Message Receiver module which constantly listens for incoming DEP messages. When a message arrives, it is handed over to the Event Factory module in order to extract its event data and event type. The Base Framework resides both on the server and the client side and contains class types for event data. Therefore, event data can be sent as serialized objects using DEP messages and be deserialized on the client side [24]. By knowing event types, the Event Factory can raise events and transfer the event data to client applications.

Besides system events, the Client Framework raises environment events, such as the network connection status, through the Environment Sensor. 


\subsection{Security}

The security model is based on the well known Kerberos protocol [30]. To realize the model, the server provides two essential services: the Authentification and the Ticket service.

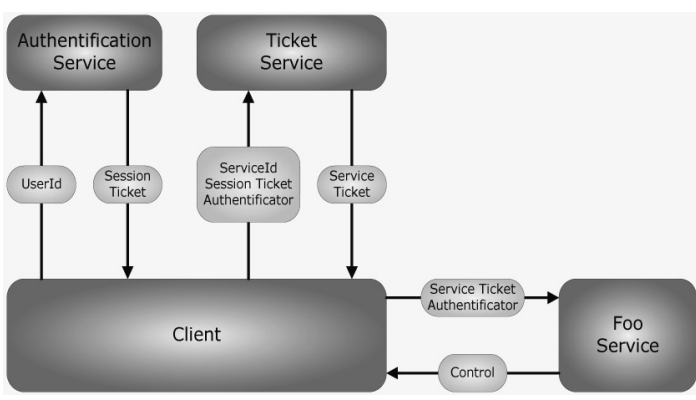

Figure 4. Authentification proces in the MILE system.

Figure 4 depicts the authorization process during the sign in procedure as well as the retrieval of a ticket from the Ticket Service needed to access other services. As soon as a user initiates sign in procedure, user identificator (e.g. user name) is sent to the Authentification Service. If the user exists, the service sends back a session ticket encrypted his or her password. If the user has given the right password, client applications will be able to decrypt the session ticket. To identify a client, the Kerberos model requires that some information in the session ticket stays encrypted, even for the client's eyes.

When a client wishes to access a service, it needs to obtain a service ticket from the Ticket Service. To achieve that, the client sends the service identificator, previously obtained session ticket and an authentificator. The authentificator contains client's identificator for the Ticket Service to be compared with the information contained in the session ticket and thus performing client identification. If all security requirements are met, the Ticket Service sends a service ticket back to the client.

By using a service ticket and an authentificator, a client can access the desired services since the services can perform client identification. In addition to that, service ticket contains a randomly generated AES key for the symmetric encryption of communication between a client and a service.

\section{MILE Applicative Modules}

MILE's applicative part consists of several modules for students' mobile devices and of an application for teachers - MClassroom (Figure 5) used to control a "virtual classroom". MClassroom is used as a replacement for a classical blackboard, for in-class real time student surveying, to keep the record of class attendance, as a repository for PowerPoint presentations with the possibility of their direct launching etc.

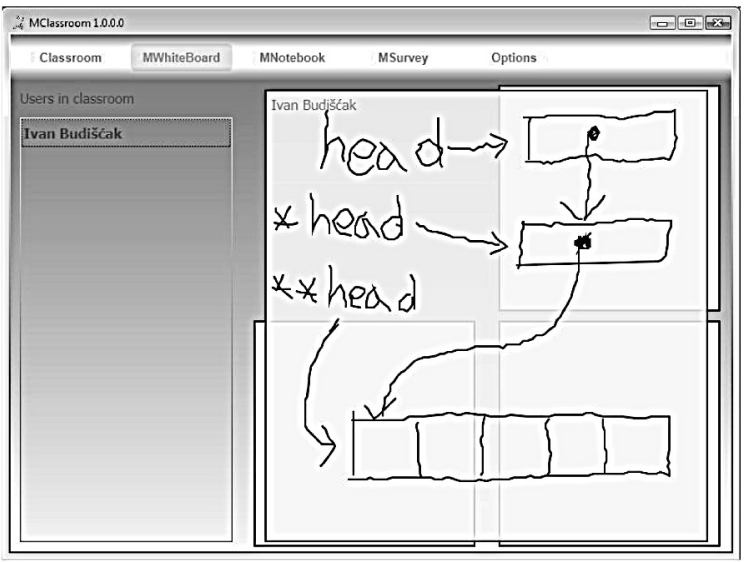

Figure 5. MILE's teacher's MClassroom application.

MILE offers many different modules (Figure 6) that support a learning environment based on mobile devices, some of the most interesting being:

- MCollaboration - used to support collaboration.

- MWhiteBoard - an alternative to classical blackboard available to mobile students and teachers.

- MNotebook - used to deliver PowerPoint presentations to mobile devices.

- MSchedule-customizable scheduler for mobile devices.

- MSurvey - instant in-class mobile assesment and surveying

- MAccessibility - accessibility features for users with special needs.

- MVirtualBoard - notice board available anytime anywhere.

- MGuide - guide to current education-related events. 
This chapter examines in detail modules that are the most important when collaborative approach to learning and blended teaching model are concerned. According to the constructivism, learning should be interactive to promote higherlevel learning and social presence [20], [27]. By using MILE modules students interact online with other students, instructors and the content.

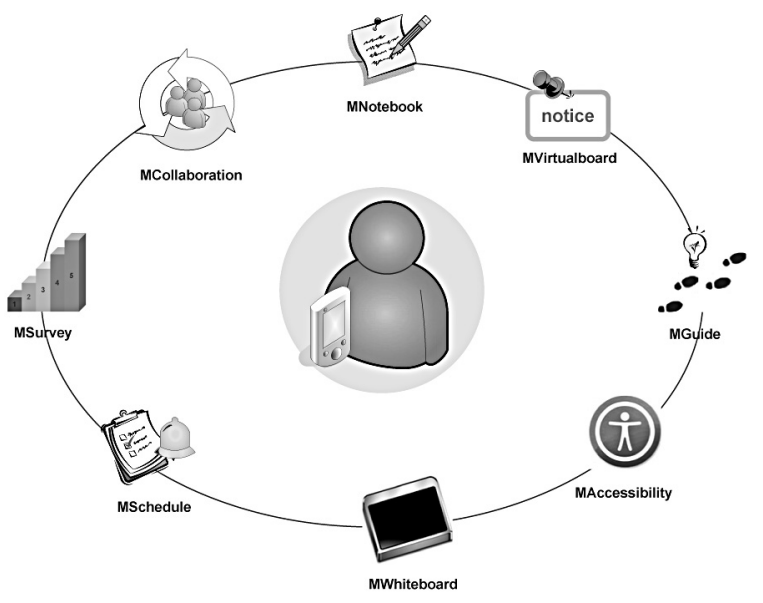

Figure 6. MILE modules.

\subsection{Collaboration as an Essential Component}

MCollaboration module (Figure 7) is primarily used to support collaboration and interaction. It can be used for online communication (chat capability for mobile users) and online collaborative group work (managed groups and users with file sharing capabilities etc.). Furthermore, it can display a map of a learning environment and locations of users within it.

In addition to that, MCollaboration is contextaware: by utilizing contextual user information provided by the server, it furthermore supports collaboration. MCollaboration's virtual map can be browsed and zoomed in to get the detailed information on colleagues' whereabouts. In this way, chance encounters and opportunities for collaboration are increased and students can easily arrange face to face meetings, thus eliminating the drawbacks of virtual communication.

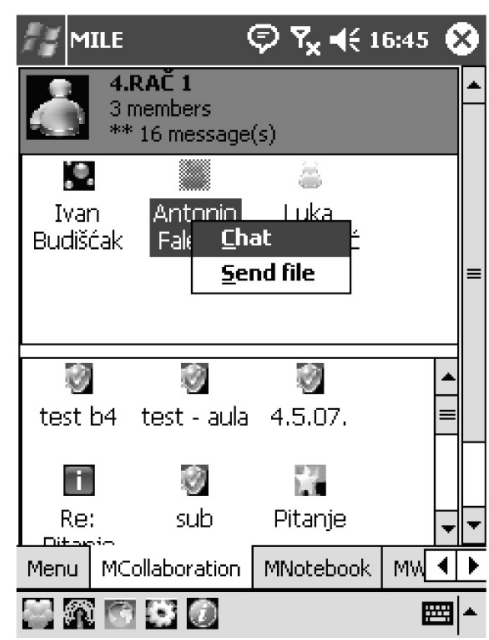

Figure 7. MCollaboration module.

\subsection{Enhanced $f 2 f$ Component of the Blended Learning Model}

Some MILE modules supplement f2f component of the blended learning model are used to support active in-class participation and real time lecture note creation, therefore enhancing student-instructor and student-content interaction.

Listening to classical lectures has always been a challenge to an average student. Reading slides, listening to a teacher, making notes in a separate notebook and synchronizing these elements after a lecture require a huge amount of time and concentration leaving less time for real learning. MILE demonstrates didactic capabilities by disseminating mobile version of teachers' presentations to students' mobile devices. Presentation on student's mobile device advances itself in synchronization with teacher's presentation. These features are enabled by MILE's contextaware and extensible architecture and present a key feature in supporting situative learning activities [40]. Similar to other mobile learning solutions, MILE could be used to support problem-based learning [40] [32], communities of practice, collaborative [21], explorative and informal learning [33].

By using MNotebook module (Figure 8) students can tag slide bullets with their own notes at any time during the presentation and save them on the server or locally on their device. After the lecture, students can access both the presentation and the notes via the MNotebook module. 
By using MClassroom application (Figure 9) teachers can access the list of students currently in a classroom. After choosing the desired ones, teacher can initiate a distributed whiteboard session. Students are able to start drawing on their mobile devices on the MWhiteboard module. The drawings are then transmitted to the MILE server and then to the teacher's MClassroom application and displayed to all students in the classroom (i.e. by using a projector).

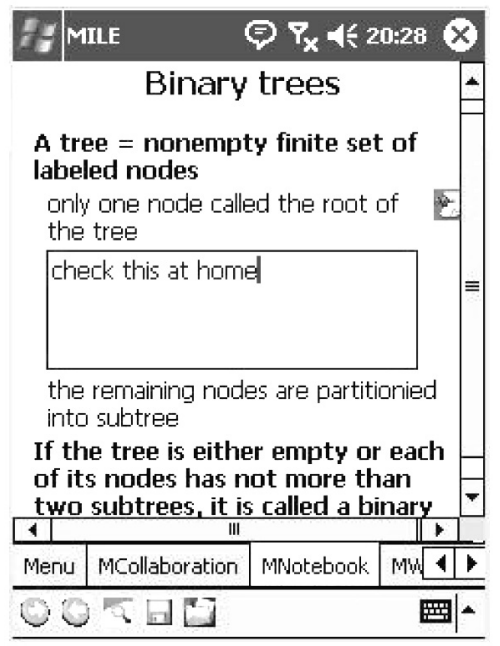

Figure 8. MNotebook module.

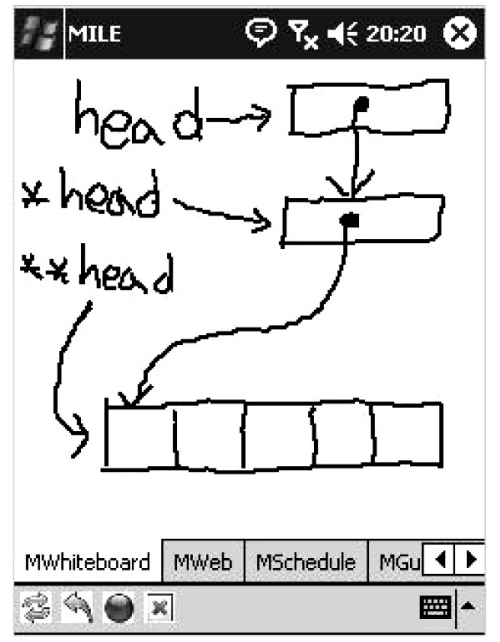

Figure 9. MWhiteboard module.

\subsection{Active Participation with Surveying}

Probably one of the most interesting things for a lecturer is to know whether his or her students are interested in the matter presented and whether they understand the lecture being held. The lack of understanding and interest can seriously affect the quality of the lecture and be a serious obstacle in transmitting the knowledge to students. By utilizing instant surveys, teachers can get information on the amount of interest and understanding students have achieved and, if needed, adjust the course and contents of lectures. By using MClassroom application (Figure 10) teachers can start a survey, and watch the votes being counted in real time. Students are presented with survey questions on their mobile devices and are required to give an answer to survey questions. Figure 10 displays the results of a typical survey question: each bar amounts to the number of students who gave a specific answer to a teacher's question.

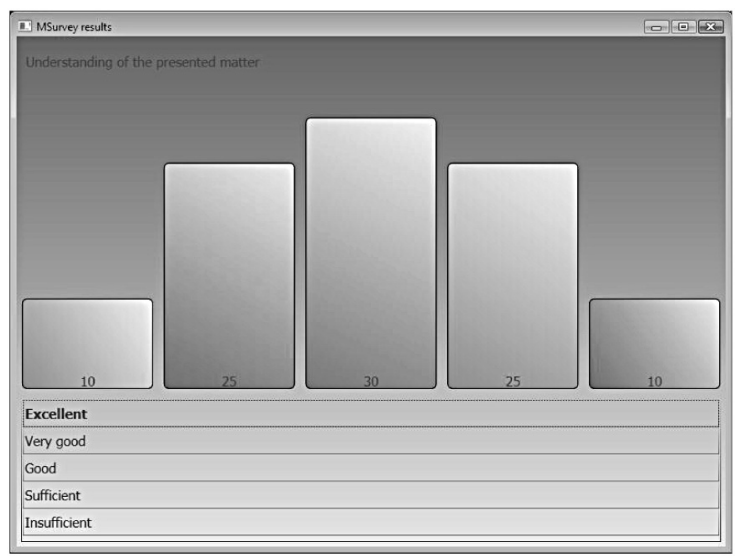

Figure 10. Survey results displayed on the MClassroom application.

\section{Experience Gained in Designing a Context-aware Mobile Learning Environment}

The development of the system started in 2004 with first attempts being described in [1]. Since then, a considerable effort has been invested in modeling and implementation of its architecture, many problems encountered and much experience gained during the process:

- The design of the distributed events protocol required synchronization mechanisms to be implemented since the UDP protocol had to be utilized for communication.

- Orientation on wireless networks presented a problem since mobile devices tend to change their IP addresses frequently. To systematically track mobile device activity and their 
current IP addresses, a variant of the SIP protocol was used.

- Additional efforts had to be invested into securing the system. For that purpose, the Kerberos protocol [30] was used. Encryption algorithms had to be carefully selected so they wouldn't use too much resources on mobile devices.

- Special care had to be given to the issues of privacy. Since MILE utilizes locationaware data, special attention was given to the users' need for privacy. Users can switch to the private mode and still utilize system's resources.

- A three-tiered architecture for the data access had to be utilized to deal with the rising system complexity.

- Applicative modules for mobile devices were implemented as self-contained and pluggable to enable their easy installation and de-installation.

- Applicative modules communicate with the rest of the system through an object-oriented programming interface in order to maximize reusability and establish firm module boundaries.

- Although the code was designed to be reusable, special implementation of the client side applicative modules had to be done for each mobile device platform used, at the same time preserving their transparent communication.

- A multidisciplinary team had to be gathered in order to provide a pedagogical perspective on one hand and to include deep technological expertise on the other.

\section{Conclusion and Further Work}

Mobile learning tries to make learning independent of the location where it happens. In that way, learning becomes more personal and less formal and better adapts to learners. Mobile learning is ambient as well. That means, it better adapts to students and the environment. The art of developing an application that better adapts to their users is called context-awareness and should be used as a first class concept when designing application for mobile clients.
The paper presented an extensible context-aware system in a form of a distributed learning network. Its name is MILE and it is primarily aimed at mobile students and supports classical and virtual learning environments. In addition to the main server component, MILE uses modules loaded on students' mobile devices to provide services to mobile users. They can collaborate, share notes, participate in whiteboard sessions etc.

The future plans include testing the system in real classroom settings, to extract information on user experience. The information will then be used to evaluate the system and the blended approach used in order to fine-tune system components. MILE will first be introduced to Programming paradigms and languages, a pregraduate course on the third year at the Faculty of Electrical Engineering and Computing. After that, depending on the user experience, the introduction to other courses will be considered.

Technically, MILE will be upgraded in several directions: new MILE modules will be created; some custom made system components will be replaced with new, out of the box solutions (e.g. WCF for mobile devices); existing modules will be further tested and technologically and graphically enhanced etc.

\section{Acknowledgments}

We would like to thank our colleagues from the Faculty of Electrical Engineering and Computing in Zagreb, Croatia who participated in the development of the MILE system: Prof. Vedran Mornar, Antonio Faletar and Luka Lovosevic.

\section{References}

[1] I. Boticki, V. MoRnAR, N. HoIC-BozIC, Introducing Location-awareness into a Learning Environment Supported by Mobile Devices. Presented in the Proceedings of the 1st International Conference on Virtual Learning, (2006) Bucharest, Romania.

[2] I. Boticki, V. Mornar, K. Andric, Supporting and enhancing teaching and learning with mobile devices. Presented in the Proceedings of the International Conference on Computer Aided Learning 2006, (2006) Villach, Austria. 
[3] P. J. Brown, The e-stick document: A framework for creating context-aware applications. Presented in the Proceedings of the Electronic Publishing, (1996) Palo Alto, California, USA.

[4] G. ChEN, D. Kotz, A Survey of Context-aware Mobile Computing Research. Technical Report TR2000-381: Department of Computer Science, Dartmouth College, Hanover, USA, 1996. www.cs. dartmouth.edu/reports/TR2000381.pdf [last accessed 14/07/2008]

[5] A. DEY, D. ABowD, Towards a better understanding of context and context-awareness. Presented in the Proceedings of the Workshop on What, Who, Where, When and How of Context-Awareness, (2000) Hague, Netherlands.

[6] S. De Freitas, M. Levene, Evaluating the development of wearable devices, personal data assistants and the use of other mobile devices in further and higher education institutions. JISC technical report, United Kindgom, 2003.

http://www.jisc.ac.uk/media/documents/ techwatch/tsw_03-05.pdf [last accessed 14/07/2008]

[7] A. Dey, D. Abowd, D. SALBER, Conceptual Framework and a Toolkit for Supporting the Rapid Prototyping of Context-aware Applications. HumanComputer Interaction (HCI) Journal, Volume 16 (2-4) (2001), 97-166.

[8] T. FAISON, Event-based Programming: Taking Events to the Limit. Apress, Berkeley, California, USA, 2006.

[9] W. G. GRISwOLd ET AL., ActiveCampus: experiments in community-oriented ubiquitous computing. Computer, Volume: 37, Issue: 10 (2004), 73-81.

[10] M. Hazas, J. ScotT, J. KrumM, Location-aware Computing Comes of Age. IEEE Computer Magazine, Vol. 37, No. 2 (2004), 95-97.

[11] A. Holzinger, A. Nischelwitzer, M. MeisenBERGER, Mobile Phones as a Challenge for $\mathrm{m}$ Learning: Examples for Mobile Interactive Learning Objects (MILOs). Presented in the Proceedings of the 3rd International Conference on Pervasive Computing and Communications Workshop (PerCom 2005 Workshops), (2005) Kauai Island, Havaii.

[12] A. Holzinger, M. ERRATH, Mobile computer Webapplication design in medicine: some research based guidelines, Universal Access in the Information Society, Volume 6 (2007), 31-41.

[13] S. HSI, H. FAIT, RFID enhances visitors' museum experience at the Exploratorium, Communications of the ACM, Vol. 48 No. 9 (2005), 60-65.

[14] P. JARVIS, J. Holford, C. GRIFFIN, The theory \& practice of learning, Kogan Page, Great Britain, 2003.

[15] Kaleidoscope Network of Excellence Mobile Learning Initiative Big Issues in Mobile Learning, Report, University of Nottingham, United Kingdom, 2006. http://mlearning.noe-kaleidoscope.org/
public/news/KALEIDOSCOPE\%20REPORT_07_ Big_Issues_In_Mobile_Learning.pdf [last accessed 14/07/2008]

[16] A. Kukulska-Hulme, Mobile Usability in Educational Contexts: What have we learnt?, The International Review of Research in Open and Distance Learning, Vol. 8, No. 2 (2007), 1-16.

[17] A. KuKulska-Hulme, J. TraXler, Mobile learning: A handbook for educators and trainers; Routledge, United Kingdom, 2005.

[18] C. MARKetT ET AL., Using short message service to encourage interactivity in the classroom. Computers and Education, Vol. 46 i3 (2006), 280-293.

[19] A. P. Massey, V. Ramesh, V. Khatri, Design, development, and assessment of mobile applications: the case for problem-based learning. IEEE Transactions on Education, Volume 49, Issue 2 (2006), 183-192.

[20] T. Mayes, S. DE Freitas, Review of e-learning theories, frameworks and models, JISC e-Learning Models Desk Study, United Kindgom, 2004. http://www . elearning . ac.uk/resources/ modelsdeskreview/ [last accessed 14/07/2008]

[21] E. M. Morken, M. Divitini, Blending Mobile and Ambient Technologies to Support Mobility in Practice-based Education: The Case of Teacher Education. Presented in the Proceedings of the MLEARN 2005 Conference, (2005) Cape Town, South Africa.

[22] L. NAISMITH ET AL., Report 11: Literature Review in Mobile Technologies and Learning, Futurelab Series, Futurelab, Bristol, United Kingdom, 2005. http://www . futurelab.org.uk/resources/ documents/lit_reviews/Mobile_Review.pdf [last accessed 14/07/2008]

[23] .NET Framework Developer Center: Windows Communication Foundation.

http://msdn2.microsoft.com/en-us/ netframework/aa663324.aspx [last accessed 14/07/2008]

[24] .NET Framework Developer Center: XML Serialization in the .NET Framework. http://msdn2.microsoft.com/en-us/ library/ms950721.aspx [last accessed 14/07/2008]

[25] OASIS Reference Model for Service-oriented Architecture. http://docs .oasis-open.org/soa-rm/v1.0/ soa-rm.pdf [last accessed 14/07/2008]

[26] J. Pascoe, N. Ryan, D. Morse, Using while moving: HCI issues in fieldwork environments. ACM Transactions on Computer-human Interaction (TOCHI), Vol. 7 No. 3 (2000), 417-437.

[27] B. Patten, I. A. Sánchez, B. Tangney, Designing collaborative, constructionist and contextual applications for handheld device, Computers \& Education, Vol. 46, Issue 3 (2006), 294-308. 
[28] O. PETERS, A pedagogical model for virtual learning spaces; Articles on flexible learning and distance education, Fernuniversitat Hagen, 1999. http://www .tbc.dk/pdf/peters-a_ pedagogicalmodel.pdf, [last accessed 14/07/2008]

[29] N. Proctor, J. Burton TATe, Modern multimedia tour pilots 2002-2003. Presented in the Proceedings of the MLEARN 2003 Conference, (2003) London, United Kingdom.

[30] RFC 4120 - The Kerberos Network Authentication Service (V5)

http://www.ietf .org/rfc/rfc4120.txt

[last accessed 14/07/2008]

[31] J. RosCHELLE, Unlocking the learning value of wireless mobile devices. Journal of Computer Assisted Learning, 19(3) (2003), 260-272.

[32] E. Sakkopoulos, M. Lytras, A. Tsakalidis, Adaptive mobile web services facilitate communication and learning Internet technologies, IEEE Transactions on Education, Vol. 49, Issue 2 (2006), 208-215.

[33] E. SCANLON, A. JoneS, J. WAYCOTT, Mobile Technologies: Prospects for Their Use in Learning in Informal Science Settings, Journal of Interactive Media in Education, Special Issue, 2005.

[34] M. SHARPLES, The design of personal mobile technologies for lifelong learning, Computers \& Education, Vol. 34 No. 3-4 (2000), 177-193.

[35] M. SharpleS, Disruptive devices: mobile technology for conversational learning, International Journal of Continuing Engineering Education and Lifelong Learning, Vol. 12, No. 5/6 (2002), 504-520.

[36] M. Sharples, J. TAYlor, G. VAVOUla, Towards a theory of mobile learning. Presented in the Proceedings of the MLEARN2005 Conference, (2005) Cape Town, Africa.

[37] O. SMORdal, J. GregORY, Knowmobile: Mobile opportunities for medical students. In Mobile Learning: A handbook for educators and trainers (A. Kukulska-Hulme and J. Traxler Eds.), (2005) pp. 99-105. Routledge, Milton Park.

[38] J. TAYLOR ET AL., Towards a task model for mobile learning: a dialectical approach. International Journal of Learning Technology, Vol. 2, No. 2/3 (2006), 138-158.

[39] K. Thorne, Blended Learning, Kogan Page, London, 2003.

[40] YuAn-KaO Wang, Context Awareness and Adaptation in Mobile Learning. Presented in the Proceedings of The 2nd IEEE International Workshop on Wireless and Mobile Technologies (WMTE'04), (2004), National Central University, Taiwan.
Received: December, 2007

Revised: July, 2008

Accepted: October, 2008

Contact addresses:

Ivica Boticki

Department of Applied Computing Faculty of Electrical Engineering and Computing

University of Zagreb

Unska 3

10000 Zagreb

Croatia

e-mail: ivica.boticki@fer.hr

Natasa Hoic-Bozic Department of Informatics

University of Rijeka Omladinska 14 51000 Rijeka Croatia

e-mail: natasah@ffri.hr

Ivan Budiscak

Department of Applied Computing Faculty of Electrical Engineering and Computing University of Zagreb Unska 3

10000 Zagreb Croatia

e-mail: ivan.budiscak@fer.hr

IVICA BOTICKI received the B.Sc. degree in computing from the University of Zagreb, Croatia, in 2004. He has been a research assistant in programming, algorithms and data structure and programming paradigms and languages at the Faculty of Electrical Engineering and Computing, University of Zagreb since 2004. His main areas of research are information systems, programming languages, e-learning, blended learning and m-learning.

NATASA HoIC-BozIC received the B.Sc. degree in mathematics and information science from the University of Rijeka, Rijeka, Croatia, in 1990 , the M. S. degree in computer and information science from the University of Ljubljana, Ljubljana, Slovenia, in 1997, and the Ph. D. degree in computing from the Faculty of Electrical Engineering and Computing (FER), University of Zagreb, Zagreb, Croatia, in 2002. She is currently an Assistant Professor in the Department of Informatics, University of Rijeka. Her main research interests include adaptive hypermedia, multimedia systems, and educational technologies, focusing on blended learning approaches.

IVAN BUDISCAK received the B.Sc. degree in computing from the University of Zagreb, Croatia, in 2008. Professional interests: information systems, distributed systems, computer graphics, mobile devices, mlearning, innovative technological solutions. 
